\title{
Clinical research assessment by flow cytometry of biomarkers for infectious stratification in an Emergency Department
}

\author{
Pénélope Bourgoin ${ }^{1,2}$, Thomas Soliveres ${ }^{3}$, Dalia Ahriz ${ }^{3}$, Isabelle Arnoux ${ }^{4}$, Christian \\ Meise $^{5,6}$, Nadine Unterwalder ${ }^{6}$, Pierre-Emmanuel Morange ${ }^{2,4}$, Pierre Michelet ${ }^{3}$, Fabrice \\ Malergue*,1 (iD) \& Thibaut Markarian ${ }^{3}$ \\ ${ }^{1}$ Department of Research \& Development, Beckman Coulter Life Sciences-Immunotech, 130 Avenue de Lattre de Tassigny, 13009 \\ Marseille, France \\ ${ }^{2}$ C2VN Department, INSERM-INRA, Aix-Marseille University, 27 Boulevard Jean Moulin, 13385 Marseille, France \\ ${ }^{3}$ Adult Emergency Department, La Timone Hospital, 264 Rue Saint Pierre, 13385 Marseille, France \\ ${ }^{4}$ Hematology Laboratory Department, La Timone Hospital, 264 Rue Saint Pierre, 13385 Marseille, France \\ ${ }^{5}$ Department of Medical Immunology, Charité - University Medicine Berlin, Augustenburger Platz 1, 13353 Berlin, Germany \\ ${ }^{6}$ Department of Immunology, Labor Berlin - Charité Vivantes GmbH, Sylter Strasse 2, 13353 Berlin, Germany \\ *Author for correspondence: fmalergue@beckman.com
}

\begin{abstract}
Aim: Management of patients with infections within the Emergency Department (ED) is challenging for practitioners, as the identification of infectious causes remains difficult with current techniques. A new combination of two biomarkers was tested with a new rapid flow cytometry technique. Materials $\&$ methods: Subjects from the ED were tested for their CD64 on neutrophils (nCD64) and CD169 on monocytes (mCD169) levels and results were compared to their clinical records. Results: Among 139 patients, 29\% had confirmed bacterial infections and 5\% viral infections. nCD64 and mCD169 respectively showed 88 and $86 \%$ sensitivity and 90 and $100 \%$ specificity for identifying subjects in bacterial or viral conditions. Conclusion: This point-of-care technique could allow better management of patients in the ED.
\end{abstract}

First draft submitted: 16 May 2019; Accepted for publication: 7 August 2019; Published online: 16 October 2019

Keywords: bacterial infections • CD169 • CD64 • emergency department • flow cytometry • viral infections

When patients are presenting with a systemic infection, practitioners in an Emergency Department (ED) are always confronted by a routine challenge to take care of them. Indeed, these pathologies could result from a large diversity of existing pathogens, such as bacteria, viruses, fungi and parasites, and even from noninfectious causes. The medical issue behind this challenge, that is discussed in this article, is the complexity of rapidly differentiating, on the basis of clinical judgment alone, the etiology of the disease between bacterial, viral and noninfectious causes, in order to trigger a correct triage and proper treatment of the patients [1].

This is especially important due to the severity of these kinds of pathologies. On the one hand, bacterial infections could evolve into a serious and potentially fatal condition such as septic shock. On the other hand, viral infections have the same acute symptoms but the risk lies in the viral spread if the virus is not specifically identified, and the patient isolated. Also, the misuse or overuse of antibiotics to treat both bacterial and viral acute febrile pathologies still remains one of the most worrying issues. Indeed, in case of empiric prescription (30-60\% of situations [2]), doctors could be confronted by either inefficiency or dangerousness of these drugs, and further contribute to the rising public health threat of increasing antimicrobial resistance [3].

The most frequently evaluated host biomarkers are CRP, white blood cell (WBC) count, polymorphonuclear (PMN) leucocyte count and PCT [4-10]. However, even if these bacterial-induced host proteins are routinely used to support diagnosis of infection, their performances are negatively affected by interpatient variability, including time from symptom onset, clinical syndrome and pathogens, which could have an impact on their sensitivity and specificity. 
Therefore, the issue arises of finding a new method of stratification for the triage and treatment of infected patients within the ED. In a recent meta-analysis, Kapasi et al. summarized published evidence on the utility of different soluble and cell-based host biomarkers to determine infection etiology, based on differences in recognition pathways and defense mechanisms triggered by bacterial or viral pathogens [11].

Of all cellular markers cited in this publication, CD64 (Fc $\gamma$-receptor-I) constitutively expressed on monocytes, but not on neutrophils, is presented as the most relevant, showing a strong correlation between increased expression on neutrophils and acute bacterial infection $[12,13]$.

Moreover, another cell surface marker, CD169 (sialoadhesin or siglec-1), has been described as overexpressed on blood monocytes early during viral infection such as in Epstein-Barr virus (EBV) enteritis and acute HIV infection [14-17].

The study hypothesis was that the combination of CD64 on neutrophils (nCD64) with CD169 on monocytes ( $m$ CD169) could be presented as markers with high sensitivities (Se) and specificities (Sp) to differentiate between bacterial and viral causes of systemic infections. In this single-center, observational, retrospective study, the aim was to evaluate their levels of expression and their performances of discrimination in subjects in the clinical settings of the adult ED of a primary University hospital in France, and to compare them to another host biomarker, the CRP. Assessment of these cell-associated markers usually requires a specialized flow cytometry laboratory, limiting their use in routine workup of patients with suspected infections. An improved and innovative flow cytometry method was used to allow their measurements on peripheral blood leucocytes in only $15 \mathrm{~min}$.

\section{Materials \& methods \\ Samples}

The study was conducted on 5 days between March and October 2017. The study population included subjects older than 18 years visiting the adult ED of La Timone University Hospital in Marseille, France during this period. This broad criterion was chosen in order to perform an observational and non-interventional retrospective study on a good proportion of subjects with various conditions.

\section{Retrospective data}

Subjects' samples were transferred to the Hematology Laboratory (HL) of La Timone Hospital. Samples were processed as part of the standard, routine clinical tests universally performed at the hospital, such as hemogram, biochemical measurements, and, if necessary, microbiological analysis. WBC and PMN counts were assessed using the Sysmex XN system (Kobe, Japan). PCT and CRP were respectively measured using the Dosage ADVIA Centaur ${ }^{\circledR}$ BRAHMS procalcitonin from Siemens (Erlangen, Germany) and the CRP Gen.3 system from COBAS (Roche, Switzerland). For the detection of viruses, blood or cerebrospinal fluids samples were examined with PCR and serum samples were tested for IgG and IgM antibodies using the LIAISON ${ }^{\circledR}$ analyzer from DiaSorin (Sallugia, Italy). For the bacterial detection, blood cultures were treated by the Brucker Mass Spectrometry system (Billerica, MA, USA), and urinary antigen tests (for Pneumococcus and Legionella), PCR (for chlamydia and mycoplasma) and/or cultures of respiratory tract secretions (nasopharyngeal swabs, tracheobronchial aspirate or bronchoalveolar lavage) were also used.

The results of the hematological and biochemical measurements of WBC, PMN, CRP and PCT, and the name of the identified pathogens if isolated, were transferred to a team of ED practitioners, blinded to the study test results. They also retrospectively recorded relevant epidemiological, clinical and paraclinical data for each subject: sex, age, clinical history (evolutionary cancer, liver disease, congestive heart failure, cerebrovascular disease, kidney disease), medical institutionalization, altered mental status, cardiac constants (systolic, diastolic and average blood pressure and pulse rate), body temperature and vital signs and symptoms (duration and kind of symptoms, prior administration of antibiotics).

Based on these electronic medical records, they then classified each subject into one of three groups:

1. Group I: subjects for which infection was ruled out. According to clinical symptoms and laboratory test results, no evidence of infection was found. This group included subjects that presented, for example, with major trauma or fractures;

2. Group II: subjects diagnosed with bacterial infections. Subjects were categorized into this group either on the basis of a positive bacterial culture result, and/or if clinical and laboratory findings such as concomitant high levels of CRP and PCT and negative viral test results strongly suggested the presence of a bacterial infection; 
3. Group III: subjects diagnosed as having viral infections. This group contained subjects that presented with typical clinical symptoms of infections, but negative bacteriological results and/or low PCT levels. In some cases, viral agents were found by antigen-based tests or serological assays.

The objective was to compare these groups, according to their medical records and routine laboratory data, to the results of flow cytometric measurement of the cell surface biomarkers.

\section{Flow cytometry assessment}

Flow cytometry assessment was performed at and with the consent and agreement of the HL of La Timone Hospital. Biomarker levels were obtained as included in the routine clinical management of subjects, at the discretion of the treating physician.

When a routine workup was finished, leftover EDTA blood tubes were pseudonymized and determination of nCD64 and mCD169 expressions in whole blood was performed according to a newly described one-step staining procedure [18].

Briefly, titrations of anti-CD169-Phycoerythrin (PE; clone 7-239) and anti-CD64-PacificBlue (PBE; clone 22), respectively custom product and product from Beckman Coulter (Beckman Coulter, Inc., CA, USA), were performed in advance in accordance with the protocol explained in the study. Then, the DURA Innovations drying process (Beckman Coulter Inc.) was applied to both antibodies, at their optimal doses, to convert the cocktail into a 'glassified' layer at the bottom of each $5 \mathrm{ml}$ testing tube. Thus, the optimized amount of each of both antibodies for a single test was present in each testing tube without error-prone pipetting of liquid reagents.

When needed, $500 \mu \mathrm{l}$ of the Versalyse lysing solution (Beckman Coulter Inc.) for the lysis of the red blood cells were added, then $5 \mu$ of EDTA blood were transferred into this tube for simultaneously staining the white blood cells.

Data were collected once on a 3-laser, 10-color Navios flow cytometer (Beckman Coulter Inc.) after only 15 min of incubation. Flow-Set beads (Beckman Coulter Inc.) were used before each analytical run in order to control a potential variability in device performance, but no harmonization between the measured values over the study period was necessary. Analysis was done using Kaluza Analysis Software (Beckman Coulter Inc.). Neutrophils and monocytes were first gated on the basis on their typical forward-and side-scatter characteristics. The expression levels of $\mathrm{nCD} 64$ and mCD169 were determined as mean of fluorescence intensities (MFI).

Practitioners at the ED and physicians of the HL were blinded for the results of flow cytometry marker analysis in order to prevent any influence on medical procedures and diagnosis.

\section{Statistical analysis}

Statistical analysis was performed using IBM SPSS Statistics version 20 (IBM SPSS, Inc., IL, USA). Quantitative data were expressed as means \pm SD or as median with 25 th and 75 th percentiles. Qualitative variables were expressed as frequency with percentages. Comparison of quantitative variables among the different groups was performed using student $\mathrm{t}$ test or Mann-Whitney U-test. Comparison of qualitative variables was performed using Fisher's exact test. Comparisons for more than two groups were performed by Freeman-Halton extension of Fisher's exact test for qualitative variables and by ANOVA or Kruskall-Wallis for quantitative variables.

Receiver operating characteristic (ROC) was used to define the best threshold of biomarker indexes. The diagnostic usefulness of nCD64 and mCD169 levels to discriminate bacterial and viral infections were investigated by means of ROC analysis. This was also applied to CRP. Analysis was based on area under curve (AUC), sensitivity $(\mathrm{Se}=\mathrm{TP} / \mathrm{P})$, specificity $(\mathrm{Sp}=\mathrm{TN} / \mathrm{N})$, positive likelihood ratio $(\mathrm{LR}+=\mathrm{Se} /[100-\mathrm{Sp}])$ and negative likelihood ratio (LR- = $[1-\mathrm{Se}] / \mathrm{Sp}$ ), with $\mathrm{P}, \mathrm{N}, \mathrm{TP}, \mathrm{TN}$, respectively corresponding to positives, negatives, true positives and true negatives patients. All values are expressed in ranges between 0 and 100, with $95 \%$ CIs. For all tests, a two-sided p-value of $<0.05$ was considered statistically significant.

\section{Results}

\section{Patients}

During the 5 days of the study period, samples of 260 patients admitted to the ED of the La Timone hospital were processed at the hospital hematology laboratory. Of these patients, 122 were lost to follow-up and excluded from further analysis because patients deliberately left the hospital before the diagnostic workup was completed and final diagnosis reached. 


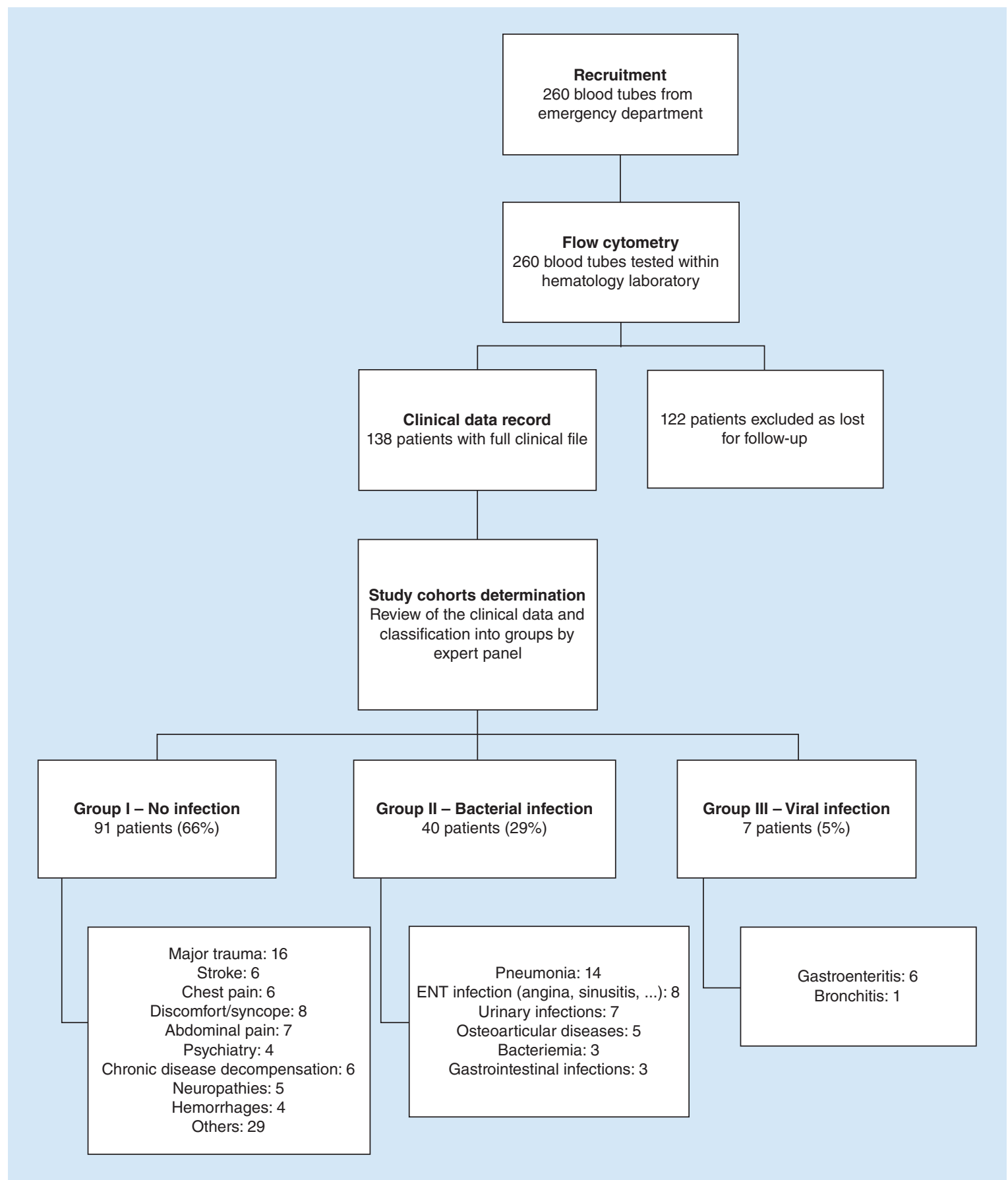

Figure 1. Study workflow: construction of the retrospective study with final numbers and diagnoses for patients who were admitted to the Emergency department.

ENT: Ears, nose and throat.

Of the remaining 138 patients, 40 (29\%) patients were classified in Group II (bacterial infection), whereas 7 $(5 \%)$ cases were classified as viral infection (Group III). The 91 (66\%) remaining patients were confirmed as having no infection (Group I). Figure 1 shows an overview of the study workflow. Supplementary Table 1 shows examples of the raw data from representative subjects. 


\begin{tabular}{|c|c|c|c|c|}
\hline Characteristic & Group I - no infection $(n=91)$ & $\begin{array}{l}\text { Group II - bacterial } \\
\text { infection }(n=40)\end{array}$ & $\begin{array}{l}\text { Group III - viral } \\
\text { infection }(n=7)\end{array}$ & p-value \\
\hline \multicolumn{5}{|l|}{ Patient sex } \\
\hline Men & $36(40 \%)$ & $20(50 \%)$ & $2(29 \%)$ & 0.582 \\
\hline Women & $55(60 \%)$ & $20(50 \%)$ & $5(71 \%)$ & \\
\hline \multicolumn{5}{|l|}{ Patient age } \\
\hline Years & $54 \pm 23$ & $66 \pm 21$ & $56 \pm 30$ & $0.022^{*}$ \\
\hline \multicolumn{5}{|l|}{ Clinical history } \\
\hline $\begin{array}{l}\text { Medical } \\
\text { institutionalization }\end{array}$ & $4(4 \%)$ & $11(28 \%)$ & $3(43 \%)$ & $\leq 0.01 *$ \\
\hline Altered mental status & $6(7 \%)$ & $12(30 \%)$ & $2(29 \%)$ & $\leq 0.01 *$ \\
\hline Evolutionary cancer & $12(13 \%)$ & $6(15 \%)$ & $1(14 \%)$ & 0.952 \\
\hline Liver disease & $4(4 \%)$ & $3(8 \%)$ & 0 & 0.683 \\
\hline $\begin{array}{l}\text { Congestive heart } \\
\text { failure }\end{array}$ & $8(9 \%)$ & $6(15 \%)$ & 0 & 0.637 \\
\hline $\begin{array}{l}\text { Cerebrovascular } \\
\text { disease }\end{array}$ & $4(4 \%)$ & $9(23 \%)$ & $2(29 \%)$ & $\leq 0.01 *$ \\
\hline Kidney disease & $7(8 \%)$ & $5(13 \%)$ & 0 & 0.754 \\
\hline \multicolumn{5}{|l|}{ Constants } \\
\hline $\begin{array}{l}\text { Systolic blood pressure } \\
(\mathrm{mmHg})\end{array}$ & $140 \pm 26$ & $132 \pm 28$ & $129 \pm 24$ & 0.383 \\
\hline $\begin{array}{l}\text { Diastolic blood } \\
\text { pressure }(\mathrm{mmHg})\end{array}$ & $75 \pm 17$ & $72 \pm 14$ & $82 \pm 17$ & 0.425 \\
\hline $\begin{array}{l}\text { Average blood } \\
\text { pressure }(\mathrm{mmHg})\end{array}$ & $96 \pm 18$ & $92 \pm 17$ & $97 \pm 18$ & 0.598 \\
\hline Pulse rate (bpm) & $82 \pm 15$ & $92 \pm 18$ & $88 \pm 20$ & $0.011^{*}$ \\
\hline Body temperature $\left({ }^{\circ} \mathrm{C}\right)$ & $37 \pm 0.3$ & $37 \pm 1$ & $38 \pm 1$ & $\leq 0.01 *$ \\
\hline
\end{tabular}

\section{Epidemiological infectious stratification}

The final cohort of 138 patients was balanced with respect to gender, as $80(58 \%)$ were women and $58(42 \%)$ were men (mean age of 57; range 22-87). Patients presented a wide range of clinical symptoms (respiratory, gastrointestinal, gynecological, neurological, urinary, ear, nose and throat $[\mathrm{ENT}]$ and cutaneous), body temperature (range 36.6-39.2), and time from symptom onset (mean 3 days). Results of univariate analysis comparing epidemiological, clinical and paraclinical data between control Group I, bacterial-infected Group II and viral-infected Group III are summarized in Table 1.

Statistically significant differences were found between groups for age (p: 0.022), medical institutionalization $(\mathrm{p}<0.001)$ and altered mental status $(\mathrm{p}<0.001)$. Indeed, relative values of these variables were higher in the bacterial group (respectively 66 years, $28 \%$ of medical institutionalization and $30 \%$ of altered mental status). Also, this group was statistically distinct from Group I and Group III for their higher number of cerebrovascular diseases $(\mathrm{p}<0.001)$ and pulse rates (p: 0.011).

All groups show statistically significant differences in their body temperatures $(\mathrm{p}<0.001)$, duration $(\mathrm{p}<0.001)$ and kind ( $\mathrm{p}<0.001)$ of symptoms, initiated antibiotherapies ( $<<0.001)$, CRP ( $<<0.001)$, and WBC (p: 0.040$)$ and PMN (p: 0.026) counts. These parameters are all constitutive of infectious definition and evolve as expected: CRP, WBC and PMN measurements rise for inflammatory bacterial and viral states, whereas PCT rises only for bacterial infections.

The most common clinical presentation associated with bacterial and viral infections were respiratory and gastrointestinal symptoms, respectively. All together, 20 pathogen species were detected (Table 4) and represented those responsible for the majority of acute infections. Escherichia coli remained the most represented pathogen. 
Table 2. Physiological and symptom data: clinical symptoms and medication recorded for the same patients.

\begin{tabular}{|c|c|c|c|c|}
\hline Characteristic & Group I - no infection $(n=91)$ & $\begin{array}{l}\text { Group II - bacterial } \\
\text { infection }(n=40)\end{array}$ & $\begin{array}{l}\text { Group III - viral } \\
\text { infection }(n=7)\end{array}$ & $p$-value \\
\hline \multicolumn{5}{|l|}{ Clinical Symptoms } \\
\hline Duration (days) & $1 \pm 3$ & $5 \pm 12$ & $3 \pm 5$ & $\leq 0.01$ * \\
\hline Respiratory & $3(3 \%)$ & $12(30 \%)$ & $2(29 \%)$ & \\
\hline Gastrointestinal & $12(14 \%)$ & $6(15 \%)$ & $4(57 \%)$ & \\
\hline Gynecological & $2(2 \%)$ & 0 & 0 & \\
\hline Neurological & $4(4 \%)$ & $1(3 \%)$ & 0 & $\leq 0.01 *$ \\
\hline Urinary & $3(3 \%)$ & $1(3 \%)$ & $1(14 \%)$ & \\
\hline Ear, nose and throat & $1(1 \%)$ & $3(7 \%)$ & 0 & \\
\hline Cutaneous & 0 & $3(7 \%)$ & 0 & \\
\hline $\begin{array}{l}\text { No infectious } \\
\text { symptoms }\end{array}$ & $66(73 \%)$ & $14(35 \%)$ & 0 & \\
\hline \multicolumn{5}{|l|}{ Antibiotherapy } \\
\hline Initiated & $7(8 \%)$ & $25(63 \%)$ & $2(29 \%)$ & $\leq 0.01 *$ \\
\hline
\end{tabular}

\begin{tabular}{|c|c|c|c|c|}
\hline Characteristic & Group I - no infection $(n=91)$ & $\begin{array}{l}\text { Group II - bacterial } \\
\text { infection }(n=40)\end{array}$ & $\begin{array}{l}\text { Group III - viral } \\
\text { infection }(n=7)\end{array}$ & p-value \\
\hline \multicolumn{5}{|l|}{$\begin{array}{l}\text { Biological } \\
\text { Measurements }\end{array}$} \\
\hline $\mathrm{CRP}(\mathrm{mg} / \mathrm{ml})$ & $7 \pm 16$ & $98 \pm 89$ & $73 \pm 104$ & $\leq 0.01$ * \\
\hline $\mathrm{PCT}(\mathrm{ng} / \mathrm{ml})$ & $0.1 \pm 0.1$ & $5.3 \pm 16.2$ & $0.84 \pm 0.8$ & 0.874 \\
\hline White blood cells (G/l) & $10 \pm 4$ & $12 \pm 6$ & $11 \pm 6$ & $0.040 *$ \\
\hline $\begin{array}{l}\text { Polymorphonuclear } \\
\text { cells (G/I) }\end{array}$ & $9 \pm 5$ & $11 \pm 6$ & $17 \pm 1$ & $0.026^{*}$ \\
\hline \multicolumn{5}{|c|}{$\begin{array}{l}\text { *p-value shows a significant difference. } \\
\text { Values are presented as either total numbers, followed by the corresponding percentages in brackets, or mean } \pm \text { standard deviation. Variables for which } \\
\text { p-values were under } \alpha=5 \% \text { were put in bold to indicate the statistically significant differences between groups. }\end{array}$} \\
\hline
\end{tabular}

\section{Table 4. Pathogens identified in the cohort study.}

Pathogen identified

Number isolated $(n=20)$

Escherichia coli

$5(25 \%)$

Klebsiella pneumoniae

$3(15 \%)$

Staphylococcus aureus

$2(10 \%)$

Proteus mirabilis

$1(5 \%)$

Enterococcus faecalis

$1(5 \%)$

Coxiella burnetii

$1(5 \%)$

Staphylococcus hominis

$1(5 \%)$

Staphylococcus haemolyticus

$1(5 \%)$

Bacteroides fragilis

$1(5 \%)$

Pseudomonas stutzeri

$1(5 \%)$

Klebsiella oxytoca

$1(5 \%)$

Hepatitis C Virus

$1(5 \%)$

Parainfluenzae

$1(5 \%)$

Numbers of bacteria and viruses isolated and/or detected in blood culture, urinary culture or serological measurements. 
(A)

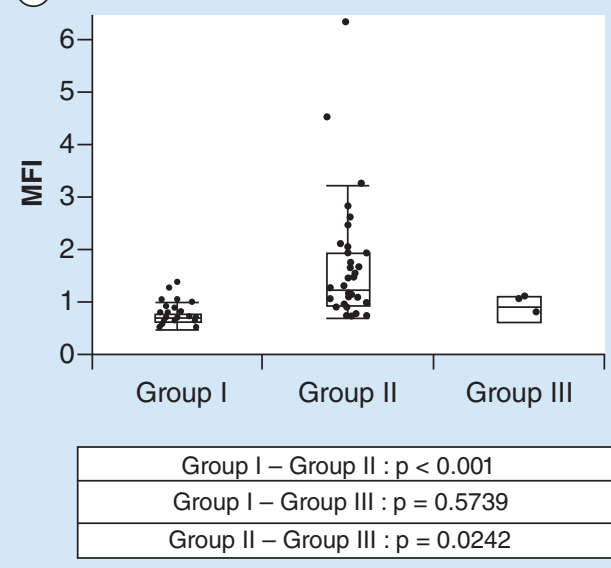

(B)

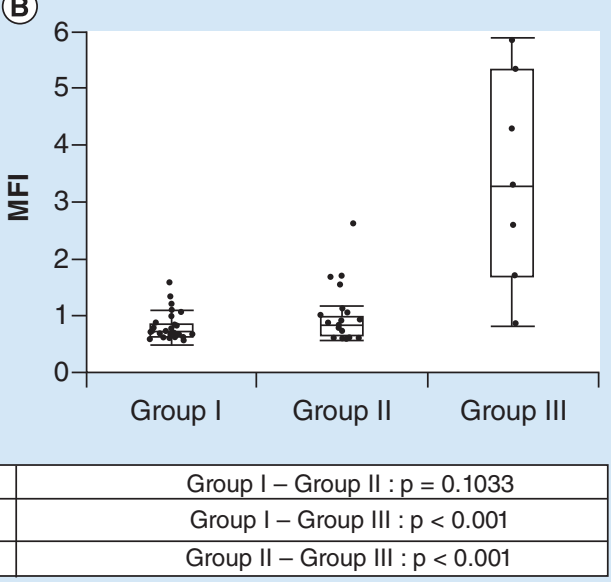

Figure 2. nCD64 and mCD169 levels for the three groups of patients. (A) CD64 expression on neutrophils for Group I (no infection), Group II (bacterial infections) and Group III (viral infections). (B) CD169 expression on monocytes for the same three groups. Data are presented as mean and standard errors. The $p$-values are calculated between each group using student $t$ test and reveal significant increase of biomarkers if $p \leq 0.05$.

MFI: Mean of fluorescence intensities.

\section{CD64 \& CD169 levels}

Figure 2A and B, respectively show nCD64 and mCD169 values for Group I, Group II and Group III.

Patients in bacterial Group II had significantly higher CD64 levels on neutrophils than patients in control Group I ( $p<0.001$ ) or viral Group III ( $p: 0.0242$ ), whereas patients in Group I and Group III presented no significant differences in $\mathrm{nCD} 64$ expression ( $\mathrm{p}: 0,5739$ ). Conversely, patients in viral Group III showed a significant increase in CD169 levels on monocytes compared with patients in control Group I $(\mathrm{p}<0.001)$ and in bacterial Group II $(\mathrm{p}<0.001)$. Patients in Group I and Group II had no significant differences of mCD169 expression (p: 0.1033).

\section{CD64 \& CD169 ROC analysis}

The optimal thresholds of each marker, for discrimination between bacterial and viral infections, have been independently assessed by ROC analysis, and their specificity and sensitivity were expressed with the respective 95\% CIs. Figure 3 presents the ROC curves of nCD64 for discrimination of patients with bacterial infections (3A), and of mCD169 for discrimination of patients with viral infections (3B), from patients of all other groups.

Using a cut-off point of $\geq 0.87$ for the Group II patients, the nCD64 MFI has a sensitivity of $87.5 \%$ (73.2-95.8), a specificity of $90.1 \%$ (82.1-95.4), a positive likelihood ratio of 8.85 (4.7-16.6) and a negative likelihood ratio of $0.14(0.06-0.3)$. The AUC is $0.93(0.87-0.97)$. However, for this marker of bacterial infections, sensitivity has to be maximal to ensure the highest proportion of positive patients in order not to miss any infectious cases. For another cut-off point of $\geq 0.66$ for the Group II patients, the nCD64 MFI has a sensitivity of 100\% (91.2-100), a specificity of 48.35\% (37.7-59.1), a LR+ of 1.94 (1.6-2.4) and a LR- of 0 . This second cut-off point seems more optimal for bacterial detection, but the lack of specificity may trigger treatment with antibiotics in some patients suspected of having a bacterial infection although not having one.

Using a cut-off point of $\geq 1.58$ for the Group III patients, the mCD169 MFI has a sensitivity of $85.71 \%$ (42.1-99.6), a specificity of $100 \%$ (96-100), a LR+ of 0 and a LR- of $0.14(0.02-0.9)$ to detect viral infections. The AUC is $0.97(0.91-0.99)$. For this marker of viral infections, specificity has to be maximal to ensure the viral etiology of the viral case, so this cut-off is optimal.

Finally, nCD64 and mCD169 levels were assessed together for their performances to discriminate between bacterial and viral infections. When using an optimal cut-off point of $\geq 0.66$ for the nCD64 MFI and an optimal cut-off point of $\geq 1.6$ for the mCD169 MFI, the combination has a sensitivity of $92.5 \%$ (80.14-97.42), a specificity of $48.35 \%$ (38.36-58.48), a positive likelihood ratio of $1.79(1.71-1.88)$ and a negative likelihood ratio of 0.15 $(0.08-0.31)$. 
(A)

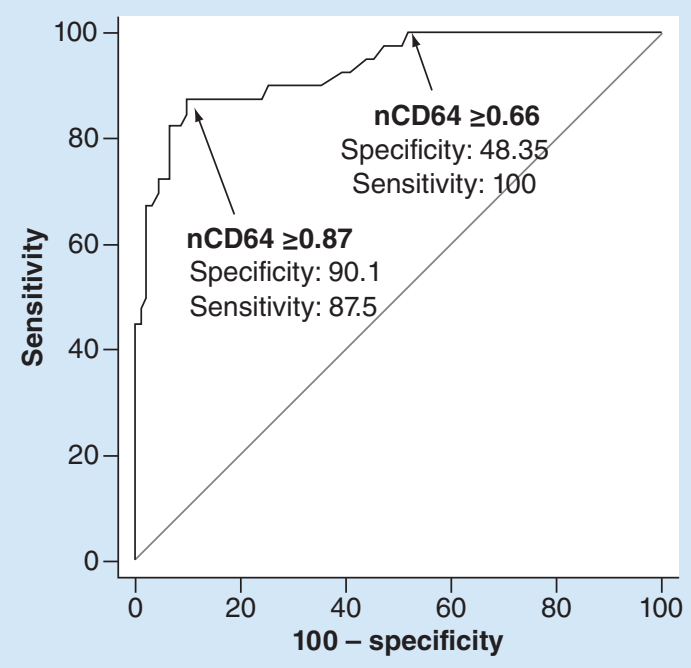

(B)

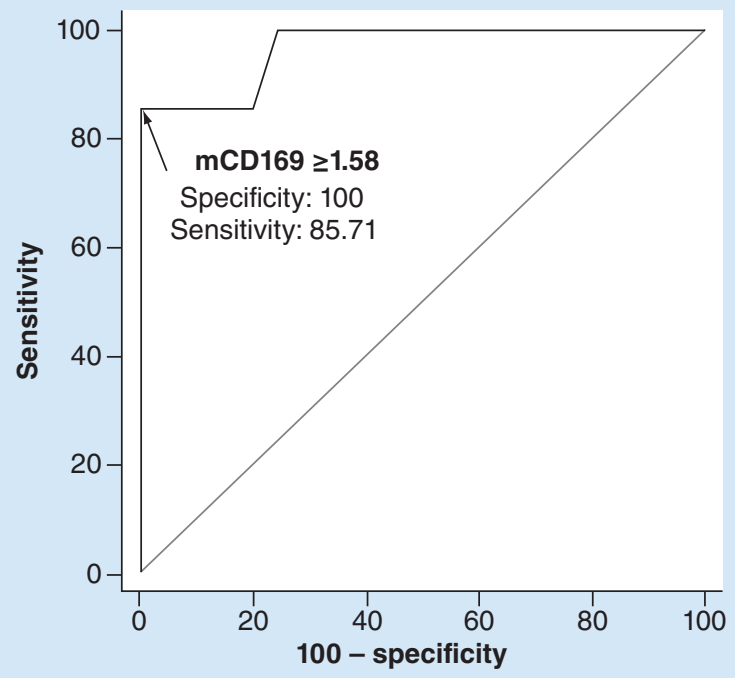

Figure 3. Receiver operating characteristic curves for nCD64 and mCD169. (A) nCD64 receiver operating characteristic curve: discrimination between presence of bacterial infection and absence of bacterial infection (no infection or viral infection). (B) mCD169 receiver operating characteristic curve: discrimination between presence of viral infection and absence of viral infection (no infection or bacterial infection).

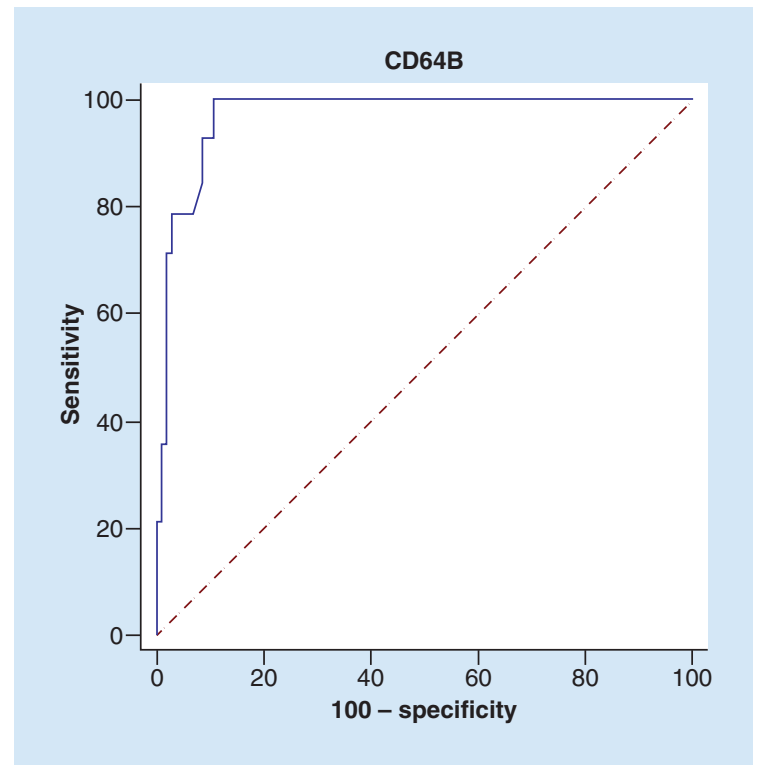

Figure 4. Receiver operating characteristic curve for nCD64 on restricted bacterial group. $\mathrm{nCD} 64$ receiver operating characteristic curve: discrimination between presence of confirmed bacterial infection (only subjects with isolated bacteria) and absence of bacterial infection (no infection or viral infection).

\section{CD64 ROC analysis when restricted to infectious-confirmed patients}

The Group II patients $(\mathrm{n}=91)$ were subjects diagnosed with bacterial infections either on the basis of a positive bacterial culture result $(\mathrm{n}=18 ; 20 \%)$, and/or if clinical and laboratory findings such as concomitant high levels of CRP and PCT and negative viral test results strongly suggested the presence of a bacterial infection $(n=73 ; 80 \%)$.

The ROC performances of nCD64 were evaluated in its capacity to discriminate patients with confirmedbacterial infection only (Group II restricted to the 18 subjects for which a positive bacterial culture result was found) from patients in all other groups (Figure 4). 
(A)

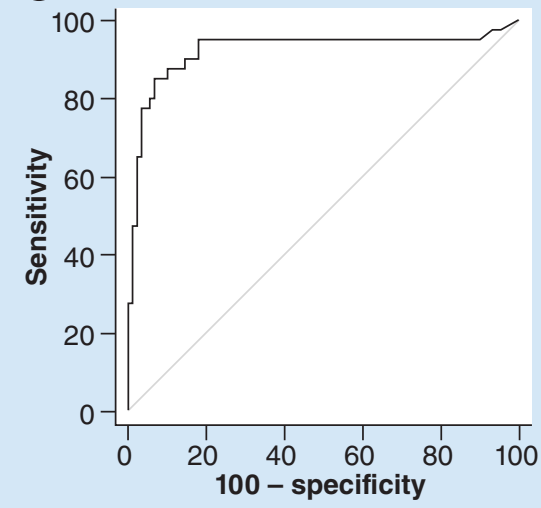

(B)

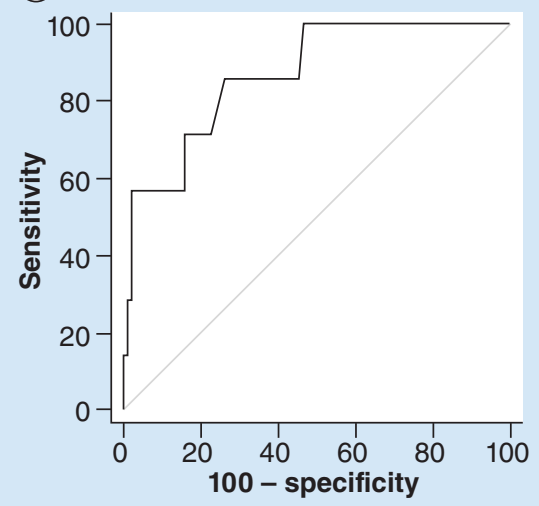

Figure 5. Receiver operating characteristic curves for CRP. (A) CRP receiver operating characteristic curve for bacterial infections: discrimination between presence of bacterial infection and absence of bacterial infection (no infection or viral infection). (B) CRP receiver operating characteristic curve for viral infections: discrimination between presence of viral infection and absence of viral infection (no infection or bacterial infection).

Using a cut-off point of $\geq 0.92$ for these restricted Group II patients, the nCD64 MFI has a sensitivity of $100 \%$ (76.8-100), a specificity of $89.4 \%$ (81.9-94.6), a LR+ of $9.45(5.4-16.5)$ and a LR- of 0 . The AUC is 0.97 $(0.92-0.99)$.

\section{CRP ROC analysis}

In comparison with both previous cellular biomarkers, Figure 5 presents the ROC curves of CRP for discrimination of patients with bacterial $(5 \mathrm{~A})$ or viral $(5 \mathrm{~B})$ infections from the other groups.

For Group II (bacterial) patients, the optimal cut-off for CRP is of $\geq 19.3$, with sensitivity of $93.18 \%$ (85.797.5), specificity of $85 \%$ (70.2-94.3), LR+ of 12.47 (5.7-27.3) and LR- of $0.16(0.08-0.3)$. The AUC is 0.92 (0.86-0.96). For Group III (viral) patients, the optimal cut-off is of $\geq 5.3$, with sensitivity of $85.71 \%$ (42.199.6), specificity of $73.86 \%$ (63.4-82.7), LR+ of $3.28(2.1-5.2)$ and LR- of $0.19(0.03-1.2)$. The AUC is 0.87 (0.78-0.93).

\section{Discussion \& conclusion}

This observational, retrospective, single-center study reports a new rapid method to distinguish the infectious etiology of acute febrile illness according to the levels of two cell surface biomarkers CD64 on neutrophils and CD169 on monocytes. To our knowledge, this is the first time these two biomarkers have been assessed in combination for differential diagnosis of bacterial and viral infections in an ED setting using a whole blood, flow cytometry assay that provides results in less than 15 min.

This study was of high importance, on the one hand, for its focus on providing good patient care in the ED, with appropriate triage of potentially infectious persons, and on the other hand, and mainly, for its focus on fighting against the rise of antimicrobial resistance due to overtreatment with antibiotics. Indeed, in case of infections, patients presenting in the ED often show obvious fever, but their other symptoms, such as cold, cough, and other urinary, abdominal, neurological or respiratory complications, cannot clearly delineate bacterial from viral infections. Even more, their clinical history and physical examinations are often not sufficient, and these issues become a routine challenge for practitioners who need to take them into account, as consequences of a bad diagnosis and therapy have been revealed as crucial for patients [19].

For years, in addition of clinical history and physical examination, bacterial culture, antigen-based immunoassay methods and other multiplexing molecular diagnostic assays have become the traditional and most specific tests for addressing the need of infectious stratification. However, they are strongly limited by their long time to results, their cost, which grows proportionally to the number of targets included, their access to the infectious site, and the extreme diversity and variability of the microbiological universe [20,21]. 
Conversely, the assessment of host biomarkers has proven to be helpful, as measurement of their levels can easily and objectively indicate potential disease activity [22,23]. The assessment of two new host cell surface biomarkers for stratification of potentially infected patients in the ED is therefore a considerable opportunity for improving sensitivity and specificity, and time-to-result, when compared with current methods. Even more, a sensitive bacterial biomarker combined with a specific viral marker could offer practitioners the ability to detect bacteria-positive cases and to differentiate viral-positive cases from negative ones.

First, the results that were achieved for the CD64 biomarker were close to those found in the literature [24-27], as its ROC curve determined an optimal nCD64 MFI threshold of $\geq 0.87$ with a sensitivity at $88 \%$ and a specificity at $90 \%$ for discriminating bacterial from nonbacterial infections. Even more, when assessed only on patients with positive bacterial culture results and not upon diagnosis, nCD64 MFI at a threshold of $\geq 0.91$ offers $100 \%$ sensitivity and $89 \%$ specificity in discriminating bacterial infections. These results were respectively equivalent or better to those found for the CRP, either in the literature [28] or in this study, as the optimal cut-off was of $\geq 19.3$ with associated sensitivity of $93 \%$ and specificity of $85 \%$. These values are also equivalent than those reported for PCT [29]. However, CD64 on neutrophils does not present some of the disadvantages of PCT and CRP. For instance, CD64 on neutrophils is not expressed in patients with inflammatory states of noninfectious origin in contrast to CRP [30]. Moreover, nCD64 has been reported to be a useful marker of localized bacterial infections, whereas PCT is mainly increased during systemic or severe invasive infections [31,32]. Finally, a large body of evidence indicates that nCD64 is a sensitive marker of early onset neonatal sepsis [33,34].

However, for stratification of bacterial infections, it is imperative to not miss any diagnosis, as doing so, without antibiotic treatment or management, increases the risk of an adverse outcome (for instance, septic shock) [35]. Minimizing the number of false negative cases is therefore necessary, even if it increases the number of false positive cases; it is therefore important to have a maximum detection sensitivity. One of the first limitations of the results concerns the sensitivity found for the nCD64, which was not as elevated as expected by the ED for a bacterial discriminator. A possibility was to decrease its cut-off to $\geq 0.66$ to achieve a sensitivity of $100 \%$, better than CRP, but with the limitation that it decreases specificity to $48.35 \%$. Another possibility would be to increase the number of tested bacterial patients only with positive bacterial culture results, as it seems that better patient selection in this group improves the nCD64 performance to the desired sensitivity of $100 \%$, while maintaining good specificity of $89 \%$. Despite these results, CD64 remains among the most powerful biomarkers for bacterial stratification, compared, for instance, to other host biomarkers such as CD35 and CD55, for which lower sensitivity of $81 \%$ and specificity of $77 \%$ was reported [36].

This is the first time that CD169 on monocytes has been evaluated in combination with another biomarker for the discrimination between viral and nonviral infections in humans. To date, CD169 had only been described on CD169-expressing splenic macrophages that have been shown to efficiently capture blood-borne pathogens and to play an important role in bridging innate and adaptive immune responses during viral infections. In HIV though, binding of sialic acid containing glycolipids to CD169 on myeloid dendritic cells has also been shown to facilitate viral transmission to $\mathrm{CD}^{+}$T-helper cells [37]. CD169 on myeloid cells such as monocytes and dendritic cells has been mainly investigated in viral pathologies in the context of pathogen recognition, in particular for HIV [38,39]. To our knowledge, assessment of monocytic CD169 for detection of viral infections and viral load has been performed only in HIV or EBV-infected humans and animal models of HIV infection [15-17]. Unpublished observations have confirmed high levels of monocytic CD169 in HIV-infected subjects with elevated viral loads, but also indicated this increase in patients with other acute viral infections, including cytomegalovirus, adenovirus and influenza virus.

Promising preliminary results have been found in this study for its use in discriminating viral from nonviral infections, as an optimal mCD169 MFI threshold of $\geq 1.6$ showed sensitivity of $86 \%$ and specificity of $100 \%$, better, for instance, than those found for the CRP, for which the optimal cut-off is of $\geq 5.3$, with equivalent sensitivity of $86 \%$ but specificity of $74 \%$. This marker seems to be well adapted to stratification of viral infections. Indeed, if a viral diagnosis is established, patients are either isolated in the hospital or sent home because more care or treatment cannot be provided. As a result, the marker must ensure no false positive cases, because in both cases referred below, medical care is particular and therefore requires a specific diagnosis. The $100 \%$ specificity of the $\mathrm{mCD} 169$, compared with the $74 \%$ of the CRP, is thus an improvement in the care of patients in the ED.

This study is promising but has several limitations. First, the study population was small. In particular, only a few patients with viral infections were included when the diagnostic performance of CD169 on monocytes was evaluated. However, the main goal was to evaluate the analytical performance of the new flow cytometry procedure 
in a proof-of-concept study in an ED setting. Second, the overall number of patients with proven bacterial or viral infections was low due to a limited microbiological workup of ED patients and the known low pathogen discovery rate in clinical samples. Hence, intensified microbiological investigations to verify the infectious cause of acute febrile illness in a larger patient cohort will be required to assess the diagnostic performance of the combination in future studies. Furthermore, it is currently unknown whether induction of nCD64 and mCD169 is equally responsive to any type of bacterial and viral infection, respectively. In addition, the influence of comorbidities and medication on the course of $\mathrm{nCD} 64$ and mCD169 expression levels during acute bacterial and viral infections needs to be thoroughly determined in order to properly interpret test results. For example, high levels of monocytic CD169 have been reported in patients with type I interferonopathies such as systemic lupus erythematosus and Sjogren syndrome during active disease $[40,41]$ potentially leading to false positive results when tested for suspected viral infections.

Finally, to these previous limitations, it could be added that this retrospective study required an analysis of clinical records retrospectively to the patient medical management. As a result, the data collection was sometimes limited, and hypothesis has been made that some patients put in the same group could present pathologies with notions of gravity and/or severity that could have been different. This may have introduced a bias in patient group formation as seen in other studies [42], instead of patients being selected in a prospective, randomized, multicentric way.

Few viral-infected patients were included, and so, combined with the nCD64, both markers discriminated patients with bacterial infections from patients who had viral infections with good sensitivity (92.5\%) but at the expense of specificity $(48.35 \%)$. These results must be supplemented with records from other virus-infected patients, but remain encouraging because biomarkers have a high negative predictive value to rule out any infection.

Moreover, simultaneous measurement of markers for bacterial and viral infections in one assay may significantly improve and expedite patient stratification and treatment decisions [43]. Although the diagnostic potential of the assay clearly needs further investigation in comparison to current standards, integration of this assay into a point-ofcare system appears possible [18]. With minimal training requirements, this application would help enable doctors to identify patients with infections in just $15 \mathrm{~min}$, on a procedure using reagents that are stable at room temperature, unitized and ready for use on only one drop of whole blood. The application would optimize the triage of patients with infectious symptoms in EDs, enable better management and faster treatment, and hence may also reduce misuse of antibiotics.

In summary, the results of this retrospective study warrant further exploration of this new and interesting combination of two biomarkers for the stratification of patients with infectious symptoms in an ED. The evaluation of $\mathrm{nCD} 64$ and mCD169 expression by flow cytometry currently discriminates between patients with bacterial infection and those with viral infection, with good values of sensitivity and specificity. Their integration in a point-of-care application that produces results in $15 \mathrm{~min}$ could ensure effective, timely triage of ED patients and improve their medical management when compared with existing methods.

\section{Summary points}

- The detection and characterization of infectious events are the major challenges for emergency medicine.

- Rapidly attributing a bacterial or viral or noninfectious origin to a febrile and/or inflammatory syndrome makes it possible to treat and refer the patient quickly.

- A new rapid diagnostic guidance solution is proposed, assessing simultaneously two biomarkers: CD64 on neutrophils (nCD64) and CD169 on monocytes (mCD169).

- Their expressions are measured within 15 min by a point-of-care procedure of flow cytometry.

- nCD64 and mCD169 levels are measured within a retrospective cohort of 139 patients in the Emergency department of La Timone Hospital, including $29 \%$ bacterial infections and $5 \%$ viral infections.

- nCD64 and mCD169, respectively, show 88 and $86 \%$ sensitivity and 90 and $100 \%$ specificity for identifying subjects in bacterial or viral conditions.

- nCD64 performances are equivalent or even better than CRP assay for which sensitivity of $93 \%$ and specificity of $85 \%$ were obtained.

- Restricting evaluation of bacterial subjects to culture confirmed cases only, nCD64 achieves even better sensitivity of $100 \%$ and specificity of $89 \%$.

- These encouraging preliminary results should be confirmed and extended through prospective and larger studies, encompassing as many infectious diseases as possible. 
Supplementary data

To view the supplementary data that accompany this paper please visit the journal website at: www.futuremedicine.com/doi/sup $\mathrm{pl} / 10.2217 / \mathrm{bmm}-2019-0142$

\section{Author contributions}

C Meisel, N Unterwalder, PE Morange, I Arnoux, T Markarian and P Michelet contributed substantially to the conception and interpretation of the study. P Bourgoin and F Malergue contributed substantially to the acquisition and analysis of flow cytometry data, while T Soliveres and D Ahriz contributed substantially to the retrieval of clinical data and interpretation. Each has participated in drafting the work or revising it. Each has provided final approval of the version to be published, and has agreed to be accountable for all aspects of the work.

\section{Acknowledgments}

The authors would like to thank the technician team from the Timone University Hospital Hematology Laboratory for their assistance in blood testing.

\section{Disclaimer}

This study was partially supported by Beckman Coulter through donations of laboratory supplies and participation of the two employees mentioned. This private company had no role in the study design, or collection and interpretation of the clinical data. Beckman Coulter and the Beckman Coulter product and service marks mentioned herein are trademarks or registered trademarks of Beckman Coulter, Inc. in the USA and other countries. All other trademarks are the property of their respective owners.

\section{Financial \& competing interests disclosure}

F Malergue and P Bourgoin are Beckman Coulter employees. P Bourgoin is recipient of a CIFRE PhD grant from the ANRT (National Agency for Research and Technology). They provided critical advice on setup of the assay and instruments and in manuscript preparation. The authors have no other relevant affiliations or financial involvement with any organization or entity with a financial interest in or financial conflict with the subject matter or materials discussed in the manuscript apart from those disclosed.

No writing assistance was utilized in the production of this manuscript.

\section{Ethical conduct of research}

The authors state that they have followed the principles outlined in the Declaration of Helsinki for all human experimental investigations. Routine care of the subjects was not modified; analyses were performed on anonymized blood left over, and all data collected in the study were part of routine clinical practice and retrieved from subject records. Results of the study had no influence on subjects' management.

\section{Data sharing statement}

The authors certify that this manuscript reports original clinical research data. Individual data that underlie the results reported in this article are available from the corresponding author following publication, including the study report and study protocol. Additional data are available upon reasonable request.

\section{References}

Papers of special note have been highlighted as: $\bullet$ of interest

1. Liang SY, Theodoro DL, Schuur JD, Marschall J. Infection prevention in the emergency department. Ann. Emerg. Med. 64(3), 299-313 (2014).

2. Ventola CL. The antibiotic resistance crisis. Pharm. Ther. 40(4), 277-283 (2015).

- Gives an overview of the antibiotic resistance issue, with a global understanding on its causes and future threats at a worldwide level.

3. Bartlett JG, Gilbert DN, Spellberg B. Seven ways to preserve the miracle of antibiotics. Clin. Infect. Dis. 56(10), 1445-1450 (2013).

4. Chalupa P, Beran O, Herwald H, Kaspř́ková N, Holub M. Evaluation of potential biomarkers for the discrimination of bacterial and viral infections. Infection 39(5), 411-417 (2011).

5. Cuquemelle E, Soulis F, Villers D et al. Can procalcitonin help identify associated bacterial infection in patients with severe influenza pneumonia? A multicentre study. Intensive Care Med. 37(5), 796-800 (2011).

6. Pfister R, Kochanek M, Leygeber T et al. Procalcitonin for diagnosis of bacterial pneumonia in critically ill patients during $2009 \mathrm{H} 1 \mathrm{~N} 1$ influenza pandemic: a prospective cohort study, systematic review and individual patient data meta-analysis. Crit. Care 18(2), R44 (2014). 
7. Nuutila J, Jalava-Karvinen P, Hohenthal U et al. A rapid flow cytometric method for distinguishing between febrile bacterial and viral infections. J. Microbiol. Methods 92(1), 64-72 (2013).

8. Haran JP, Beaudoin FL, Suner S, Lu S. C-reactive protein as predictor of bacterial infection among patients with an influenza-like illness. Am. J. Emerg. Med. 31(1), 137-144 (2013).

9. Sklavou R, Karavanaki K, Critselis E et al. Variation of serum C-reactive protein (CRP) over time in pediatric cancer patients with febrile illness and its relevance to identified pathogen. Clin. Biochem. 45(15), 1178-1182 (2012).

10. Weh J, Antoni C, Weiß C, Findeisen P, Ebert M, Böcker U. Discriminatory potential of C-reactive protein, cytokines, and fecal markers in infectious gastroenteritis in adults. Diagn. Microbiol. Infect. Dis. 77(1), 79-84 (2013).

11. Kapasi AJ, Dittrich S, González IJ, Rodwell TC. Host biomarkers for distinguishing bacterial from non-bacterial causes of acute febrile illness: a comprehensive review. PLoS ONE 11(8), e0160278 (2016).

- This comprehensive review summarizes the diagnostic performances of host biomarkers capable of differentiating bacterial from nonbacterial infections to guide use of antibiotics.

12. Icardi M, Erickson Y, Kilborn S, Stewart B, Grief B, Scharnweber G. CD64 index provides simple and predictive testing for detection and monitoring of sepsis and bacterial infection in hospital patients. J. Clin. Microbiol. 47(12), 3914-3919 (2009).

13. Hoffmann JJML. Neutrophil CD64: a diagnostic marker for infection and sepsis. Clin. Chem. Lab. Med. 47(8), 903-916 ( 2009).

14. van der Kuyl AC, van den Burg R, Zorgdrager F, Groot F, Berkhout B, Cornelissen M. Sialoadhesin (CD169) expression in CD14 ${ }^{+}$ cells is upregulated early after HIV-1 infection and increases during disease progression. PLoS ONE 2(2), e257 (2007).

15. Kim W-K, McGary CM, Holder GE et al. Increased expression of CD169 on blood monocytes and its regulation by virus and CD8 T cells in Macaque Models of HIV infection and AIDS. AIDS Res. Hum. Retrov. 31(7), 696-706 (2015).

- This study precisely demonstrates that CD169 could be a biomarker of HIV infection, in macaques.

16. Rempel H, Calosing C, Sun B, Pulliam L. Sialoadhesin expressed on IFN-induced monocytes binds HIV-1 and enhances infectivity. PLoS ONE 3(4), e1967 (2008).

17. Ashokkumar C, Gabriellan A, Ningappa M, Mazariegos G, Sun Q, Sindhi R. Increased monocyte expression of sialoadhesin during acute cellular rejection and other enteritides after intestine transplantation in children. Transplantation 93(5), 561-564 (2012).

- Indicates that CD169 could be a biomarker of HIV and EBV infections, in humans.

18. Bourgoin P, Hayman J, Rimmelé T, Venet F, Malergue F, Monneret G. A novel one-step extracellular staining for flow cytometry: proof-of-concept on sepsis-related biomarkers. J. Immunol. Methods 470, 59-63 (2019).

- This proof-of-concept study introduces the new one-step procedure for flow cytometry, feasible at the point-of-care and giving results in less than $15 \mathrm{~min}$.

19. Ray P, Birolleau S, Lefort $Y$ et al. Acute respiratory failure in the elderly: etiology, emergency diagnosis and prognosis. Crit. Care 10(3), R82 (2006).

20. Laxminarayan R, Duse A, Wattal C et al. Antibiotic resistance - the need for global solutions. Lancet Infect. Dis. 13(12), 1057-1098 (2013).

21. Parrott G, Kinjo T, Nabeya D et al. Evaluation of Anyplex ${ }^{\top M}$ II RV16 and RB5 real-time RT-PCR compared to Seeplex ${ }^{\circledR}$ RV15 OneStep ACE and PneumoBacter ACE for the simultaneous detection of upper respiratory pathogens. J. Infect. Chemother. 23(12), 859-861 (2017).

22. Oved $\mathrm{K}$, Cohen A, Boico O et al. A novel host-proteome signature for distinguishing between acute bacterial and viral infections. PLoS ONE 10(3), e0120012 (2015).

23. Srugo I, Klein A, Stein M et al. Validation of a novel assay to distinguish bacterial and viral infections. Pediatrics 140(4), e20163453 (2017).

24. Li S, Huang X, Chen Z et al. Neutrophil CD64 expression as a biomarker in the early diagnosis of bacterial infection: a meta-analysis. Int. J. Infect. Dis. 17(1), e12-e23 (2013).

- This meta-analysis reveals that nCD64 is a promising biomarker for diagnosing bacterial infections.

25. Gibot S, Béné MC, Noel R et al. Combination biomarkers to diagnose sepsis in the critically Ill patient. Am. J. Respir. Crit. Care Med. 186(1), 65-71 (2012).

26. Mokuda S, Doi O, Takasugi K. Simultaneous quantitative analysis of the expression of CD64 and CD35 on neutrophils as markers to differentiate between bacterial and viral infections in patients with rheumatoid arthritis. Mod. Rheumatol. 22(5), $750-757$ (2012).

27. Cid J, Aguinaco R, Sánchez R, García-Pardo G, Llorente A. Neutrophil CD64 expression as marker of bacterial infection: a systematic review and meta-analysis. J. Infect. 60(5), 313-319 (2010).

28. Xiong SD, Pu LF, Wang HP et al. Neutrophil CD64 Index as a superior biomarker for early diagnosis of infection in febrile patients in the hematology department. Clin. Chem. Lab. Med. 55(1), 82-90 (2017).

- Demonstrates the clinical performances of the neutrophil CD64 index for early identification of infections in febrile patients, in comparison or in combination to other reference biomarkers. 
29. Xu N, Chen J, Chang X et al. nCD64 index as a prognostic biomarker for mortality in acute exacerbation of chronic obstructive pulmonary disease. Ann. Saudi Med. 36(1), 37-41 (2016).

30. Markic J, Jeroncic A, Polancec D et al. CD15s is a potential biomarker of serious bacterial infection in infants admitted to hospital. Eur. J. Pediatr. 172(10), 1363-1369 (2013).

31. Meisner M. Update on procalcitonin measurements. Ann. Lab. Med. 34(4), 263-273 (2014).

32. Gilbert DN. Role of procalcitonin in the management of infected patients in the intensive care unit. Infect. Dis. Clin. North Am. 31(3), 435-453 (2017).

33. Tang Z, Qin D, Tao M et al. Examining the utility of the CD64 index compared with other conventional indices for early diagnosis of neonatal infection. Sci. Rep. 8(1), 9994 (2018).

34. Ng PC, Li G, Chui KM et al. Neutrophil CD64 is a sensitive diagnostic marker for early-onset neonatal infection. Pediatr. Res. 56(5), 796-803 (2004).

35. Vijayan AL, Vanimaya, Ravindran $S$ et al. Procalcitonin: a promising diagnostic marker for sepsis and antibiotic therapy. J. Intensive Care 5(1), 1-7 (2017).

36. Nuutila J, Jalava-Karvinen P, Hohenthal U et al. Use of complement regulators, CD35, CD46, CD55, and CD59, on leukocytes as markers for diagnosis of viral and bacterial infections. Hum. Immunol. 74(5), 522-530 (2013).

37. Pino M, Erkizia I, Benet $S$ et al. HIV-1 immune activation induces Siglec-1 expression and enhances viral trans-infection in blood and tissue myeloid cells. Retrovirology 12(1), 37 (2015).

38. Zou Z, Chastain A, Moir S et al. Siglecs facilitate HIV-1 infection of macrophages through adhesion with viral sialic acids. PLoS ONE 6(9), e24559 (2011).

39. Izquierdo-Useros N, Lorizate M, Puertas MC et al. Siglec-1 is a novel dendritic cell receptor that mediates HIV-1 trans-infection through recognition of viral membrane gangliosides. PLoS Biol. 10(12), e1001448 (2012).

40. Rose T, Grützkau A, Hirseland $\mathrm{H}$ et al. IFN $\alpha$ and its response proteins, IP-10 and SIGLEC-1, are biomarkers of disease activity in systemic lupus erythematosus. Ann. Rheum. Dis. 72(10), 1639-1645 (2013).

41. Lisney AR, Szelinski F, Reiter K, Burmester GR, Rose T, Dörner T. High maternal expression of SIGLEC1 on monocytes as a surrogate marker of a type I interferon signature is a risk factor for the development of autoimmune congenital heart block. Ann. Rheum. Dis. 76(8), 1476-1480 (2017).

42. van Houten $\mathrm{CB}$, de Groot JAH, Klein A et al. A host-protein based assay to differentiate between bacterial and viral infections in preschool children (OPPORTUNITY): a double-blind, multicentre, validation study. Lancet Infect. Dis. 17(4), 431-440 (2017).

43. Dittrich S, Tadesse BT, Moussy F et al. Target product profile for a diagnostic assay to differentiate between bacterial and non-bacterial infections and reduce antimicrobial overuse in resource-limited settings: an expert consensus. PLoS ONE 11(8), e0161721 (2016).

- This expert consensus defines a target product profile with associated requirements for a bacterial versus nonbacterial assay to guide future products' developments. 